\title{
Nueva clasificación clínica de la infección por virus Dengue: ¿qué tan útil es en áreas endémicas?
}

\section{New clinical classification of dengue virus infection: how helpful is it in endemic areas?}

\section{Nova classificação clínica da infecção pelo vírus da dengue: qual é a utilidade em áreas endêmicas?}

\author{
Teresa Isabel Véliz-Castro ${ }^{\mathrm{I}}$ \\ teresa.veliz@unesum.edu.ec \\ Nereida Valero-Cedeño ${ }^{\text {II }}$ \\ nereida.valero@unesum.edu.ec \\ Violeta Maricela Dalgo-Flores III \\ violeta.dalgo@espoch.edu.ec \\ Maritza Guadalupe Cabrera-Hernández IV \\ mcabrera@ucm.cl \\ María José Pinos-Cedeño ${ }^{\mathrm{V}}$ \\ masepc@gmail.com
}

\author{
Anyelo Alberto Duran-Mojica VI \\ anyeloduran@gmail.com
}

Luis Eduardo Gallardo-Villasmil VII

luisgallardo10692@hotmail.com

Víctor Saldaña-Núñez VIII

saldananunezvictor@gmail.com

Mariana Torres-Portillo IX

marianatorresp@hotmail.com

Recibido: 10 de marzo de 2019 *Aceptado: 08 de mayo de 2019 * Publicado: 05 de julio de 2019

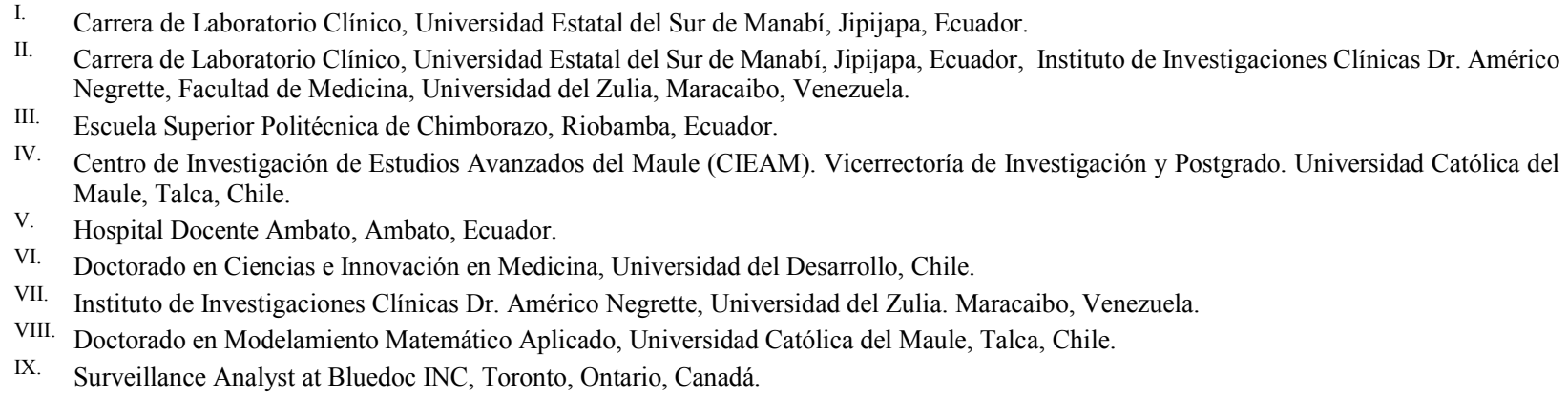




\title{
Resumen
}

La infección por el virus Dengue es considerada hoy día una emergencia epidemiológica, debido al incremento de áreas geográficas endémicas y a los cambios en el patrón de la infección, incrementando el riesgo de morbimortalidad. El Dengue tiene un espectro de severidad variado, con evolución clínica y resultados impredecibles. La mayoría de las infecciones presentan un cuadro clínico autoresolutivo, pero otros resultan en formas graves de la enfermedad. Las limitaciones del esquema de clasificación de la Organización Mundial de la Salud de 1997 han sido tema de discusión. Una afirmación, es que el dengue hemorrágico y el síndrome de choque por dengue equivalen a formas graves de la enfermedad. Sin embargo, a partir de numerosos estudios, se ha revelado una significativa proporción de casos clínicamente graves que se encuentran en la clasificación de Fiebre Dengue, lo cual ha ocasionado manejo inadecuado y desmejoras en el pronóstico de los pacientes. La clasificación de 2009, propone la detección oportuna de pacientes en riesgo de evolucionar a dengue grave, sin embargo, persisten dificultades para la clasificación. En el presente artículo se plantea analizar estos criterios y su aplicación en países endémicos como Venezuela, donde pudieran generarse demanda de hospitalizaciones ante un signo de alarma en un paciente que pudiera manejarse de forma ambulatoria, o que haya alguna manifestación inusual aún no especificada. Se propone con este esbozo caracterizar un complejo sindromático de complicaciones en dengue, descripción y documentación de las mismas, con el propósito de optimizar el abordaje clínico del paciente.

Palabras claves: Dengue; Clasificación; Clínica; Severidad.

\begin{abstract}
Dengue virus infection is considered today an epidemiological emergency due to increased endemic geographic areas and changes in the pattern of infection, with higher risk of morbidity and mortality. Dengue has a varied spectrum of severity, with clinical evolution and unpredictable results. Most infections have an autoresolutive outcome, but others result in severe forms of the disease. The limitations of the classification scheme of the World Health Organization in 1997 have been discussed. One claim is that hemorrhagic dengue and dengue shock syndrome are severe forms of the disease. However, from numerous studies, it has been revealed a significant proportion of severe cases named in the classification as Dengue Fever, which has led to mismanagement and deteriorations in the

4 Vol. 5, núm. 3, julio 2019, pp. 3-26

4 Teresa Isabel Véliz Castro, Nereida Valero Cedeño, Violeta Maricela Dalgo Flores, Maritza Guadalupe Cabrera Hernández, María José Pinos Cedeño, Anyelo Alberto Duran Mojica, Luis Eduardo Gallardo Villasmil, Víctor Saldaña Núñez; Mariana Torres Portillo
\end{abstract}


prognosis of patients. The classification of 2009, proposes the timely detection of patients at risk for progression to severe dengue, however difficulties persist for classification. In this review we want to analyze these criteria and their application in endemic countries such as Venezuela, where demand for hospitalization may arise before a warning sign in a patient could be managed on an outpatient basis, or has some unusual manifestation yet unspecified. This approach is proposed to characterize a symptom complex of complications in dengue, description and documentation of the same, in order to optimize the clinical management of the patient.

Key words: Dengue; Classification; Clinic; Severity.

\section{Resumo}

A infecção pelo vírus da dengue é hoje considerada uma emergência epidemiológica, devido ao aumento de áreas geográficas endêmicas e mudanças no padrão de infecção, aumentando o risco de morbidade e mortalidade. A dengue tem um espectro de gravidade variada, com evolução clínica e resultados imprevisíveis. A maioria das infecções apresenta um quadro clínico autodestrutivo, mas outras resultam em formas graves da doença. As limitações do esquema de classificação da Organização Mundial da Saúde de 1997 têm sido objeto de discussão. Uma alegação é que a febre hemorrágica da dengue e a síndrome do choque da dengue são formas graves da doença. No entanto, a partir de inúmeros estudos, uma proporção significativa de casos clinicamente graves encontrados na classificação da Dengue foi revelada, o que causou um manejo inadequado e uma deterioração no prognóstico dos pacientes. A classificação de 2009 propõe a detecção oportuna de pacientes com risco de desenvolver dengue grave, no entanto, as dificuldades de classificação persistem. Neste artigo, queremos analisar esses critérios ea sua aplicação em países endêmicos como a Venezuela, onde poderiam gerar demanda por internações antes de um sinal de alerta em um paciente poderia ser tratado em nível ambulatorial, ou tem alguma manifestação incomum ainda não especificada. Propõe-

5 Vol. 5, núm. 3, julio 2019, pp. 3-26

Teresa Isabel Véliz Castro, Nereida Valero Cedeño, Violeta Maricela Dalgo Flores, Maritza Guadalupe Cabrera Hernández, María José Pinos Cedeño, Anyelo Alberto Duran Mojica, Luis Eduardo Gallardo Villasmil, Víctor Saldaña Núñez; Mariana Torres Portillo 
se com este esquema caracterizar um complexo sindromático de complicações da dengue, descrição e documentação das mesmas, com o objetivo de otimizar a abordagem clínica do paciente.

Palavras chave: Dengue; Classificação; Clínica; Gravidade.

\section{Introducción.}

El dengue (DEN) es una enfermedad viral, de carácter endemo-epidémico, transmitida por la picadura de mosquitos hembras del género Aedes, principalmente Aedes aegypti (A. aegypti). Actualmente es la arbovirosis de mayor relevancia a nivel mundial en términos de morbilidad, mortalidad y afectación económica $(1,2,3,4,5)$.

El virus dengue (DENV), está constituido por ARN genómico de sentido positivo, con una cadena sencilla de aproximadamente $10,7 \mathrm{~kb}$ de longitud, rodeado por una nucleocápside de simetría icosahédrica, de $30 \mathrm{~nm}$ de diámetro, la cual está constituida por una proteína $\mathrm{C}$ (cápside) de $11 \mathrm{kd}$. Esta estructura se encuentra rodeada por una bicapa lipídica de $10 \mathrm{~nm}$ de grosor; en la que se encuentran insertadas las proteínas estructurales $\mathrm{E}$ que conforma la envoltura y $\mathrm{M}$ que forma la membrana del virus, dando lugar a proyecciones que sobresalen de la superficie de los viriones. El virión completo mide alrededor de $50 \mathrm{~nm}$ de diámetro, y tiene forma esférica. El ARN de las partículas virales maduras codifica para una poliproteína, que es posteriormente procesada por enzimas tanto del virus como del hospedador, dando lugar a tres proteínas estructurales (prM/M, E y C) y siete no estructurales (NS1, NS2A, NS2B, NS3, NS4A, NS4B, NS5) $(6,7,8)$. Actualmente existen 4 serotipos antigénicamente relacionados (DENV-1, DENV-2, DENV-3, DENV-4) y un quinto serotipo sin actividad demostrada en humanos (DENV5), los cuales exhiben secuencias de aminoácidos idénticas en aproximadamente 70\%. Pertenece a la familia Flaviviridae, del género Flavivirus (del latín flavus,

\footnotetext{
6 Vol. 5, núm. 3, julio 2019, pp. 3-26 
amarillo), recibe este nombre por tener como miembro característico de la familia al virus de la fiebre amarilla; incluye un grupo de más de 68 agentes virales transmitidos por artrópodos y de los cuales por lo menos 55\% están asociados con alguna enfermedad en el hombre (8-14).

La incidencia y epidemias de dengue han aumentado exponencialmente en los últimos 50 años a escala mundial. Se estima que de los 2500 millones de personas que viven en áreas endémicas, 50 millones se infectan anualmente y más de 500000 contraen su forma más grave $(15,23)$. Actualmente, el DENV es endémico en más de 100 países del Sudeste Asiático, el Pacífico Occidental, América, África y el Medio Oriente. Durante las epidemias, las tasas de ataque pueden llegar a afectar a 80$90 \%$ de las personas susceptibles 20 y la letalidad puede ser mayor de 5\% $(12,13)$.

El dengue en América adquiere cada vez mayor importancia como enfermedad re-emergente debido a la co-circulación de los cuatro serotipos del virus, al incremento en número de casos y consecuentemente a la expansión de áreas epidémicas y a la aparición de casos graves $(20,21,24)$. Durante las últimas décadas, en Las Américas se registra el más drástico incremento en la actividad del dengue, sostenible en los últimos 25 años, con brotes epidémicos que se repiten de manera cíclica especialmente en Brasil, Colombia, Cuba, Ecuador, Perú, Venezuela y Paraguay: la situación epidemiológica del dengue es de alta complejidad, lo que obliga a redoblar los esfuerzos para la implementación de una estrategia de gestión integrada (14).

En Venezuela, ha permanecido en situación de endemicidad en muchas regiones del país, produciendo grandes epidemias y causando importante impacto socio-económico en la población (15). En 2009, el Ministerio de Salud de Venezuela reportó un total de 35.546 casos sospechosos de dengue y 2.782 dengues hemorrágicos y el último anuario publicado por el mismo organismo correspondiente 
al año 2011, se registran, 30209 casos de fiebre dengue y 1420 casos de dengue hemorrágico (16). En 2013, se reportan 43.964 casos de dengue en Venezuela. Si se le compara con el mismo período en 2012, cuando se reportaron 34.393 contagios, el aumento fue de $27 \%$ en un año $(17,18)$.

En el estado Zulia, han ocurrido epidemias periódicas durante los últimos 15 años y las cifras de morbilidad entre epidemia han sido de hasta 3000 casos anuales (19). En 2011, se recogen 4140 casos de fiebre dengue y 194 de los hemorrágicos en el mismo Estado (16). En 2014, el estado Zulia ocupa el cuarto lugar, con 3.643 casos de dengue (17). A esto, contribuyen, entre otros factores, las particularidades climáticas de la región, como sus elevadas temperaturas, niveles de precipitación y la humedad relativa y la humedad relativa $(14,18)$. El Estado Zulia históricamente ha sido afectado por epidemias de Dengue. Esta enfermedad afecta diferentes regiones del continente, cuyo agente etiológico tiene antecedente de circulación en la zona debido, entre otras causas, a la proliferación de su vector principal Aedes aegypti. A un total de 8.279 sueros de pacientes con clínica de infección por virus dengue, se les determinaron anticuerpos $\operatorname{IgM}(n=8.279)$ e $\operatorname{IgG}$ anti-dengue $(n=196)$ por Elisa de captura, el aislamiento viral se realizó en cultivo de células C6/36 y la identificación por Inmunofluorescencia Indirecta. El estudio arrojó 70,32\% de seropositividad (5.822/8.279), homogéneamente distribuida entre los grupos, con mayor gravedad en los adolescentes, adultos jóvenes y tercera edad con circulación de los serotipos DENV-3 y 4. Se encontró una frecuencia mayor $(\mathrm{p}<0,0001)$ en los casos de Fiebre Dengue (FD) para el 2009 y 2010. No hubo diferencias significativas en cuanto al sexo. Las infecciones secundarias (IS) fueron de 48,98\%. Estos hallazgos evidencian la alta incidencia de dengue en el Zulia, confirmándose el carácter hiperendémico dado la presencia de muchos de los factores de riesgo detonantes a la ocurrencia de infección por este virus $(18,19)$.

8 Vol. 5, núm. 3, julio 2019, pp. 3-26 
Un estudio reciente, realizado en el estado Zulia (18), utilizaron por vez primera información disponible a escala espacial y temporal con variables tanto climáticas, como no climáticas. Como variables climáticas analizadas, se incluyen temperatura, precipitación y el fenómeno global del Niño, a través de un enfoque de Modelo Aditivo Mixto Generalizado. Este estudio fue beneficiado del uso de data climática remota satelital suministrada por la Agencia Internacional NASA para fines científicos. Mientras las variables no climáticas incluyeron información demográfica, geográfica y socioeconómica. En dicho estudio, una oscilación multianual fue confirmada en el estado Zulia durante el periodo 2002-2008, registrando un mayor índice durante 2007. Un análisis exploratorio, demostró con los casos totales de dengue en el estado de Zulia, durante 2008 hasta 2016 suministrados por el Ministerio de Salud de la región; para investigar patrones en la evolución de la dinámica de la enfermedad durante este nuevo periodo: Como categoría principal, se consideró la segregación anual y posteriormente una segregación mensual y semanal de los casos de dengue en el estado de Zulia en los 21 municipios que conforman la región. Cabe mencionar que desde el año 2013, el municipio de Páez no aparece incluido en los registros presentados en la base de datos suministrada por la entidad regional; sin embargo, la data corresponde al nuevo municipio denominado Goajira, el cual considera el mismo territorio.

Otra observación importante en relación a la organización de la data, es que las semanas fueron ordenadas siguiendo el criterio de funcionamiento de oficinas; es decir de lunes a viernes. Por tal razón, todo mes terminado en día miércoles en adelante, su registro corresponde al mes actual; en caso contrario pasa como registro del siguiente mes. Adicionalmente, tal como se mencionó, el presente estudio considera los casos totales de dengue. Sin embargo, aun cuando durante el periodo 2008-2013 la clasificación del dengue solo incluía dos categorías (Clásico y Hemorrágico); para el periodo 2014-

\footnotetext{
9 Vol. 5, núm. 3, julio 2019, pp. 3-26

9 Teresa Isabel Véliz Castro, Nereida Valero Cedeño, Violeta Maricela Dalgo Flores, Maritza Guadalupe Cabrera Hernández, María José Pinos Cedeño, Anyelo Alberto Duran Mojica, Luis Eduardo Gallardo Villasmil, Víctor Saldaña Núñez; Mariana Torres Portillo
} 
2016 entro en vigencia la nueva clasificación, a saber: DSSA, DCSA y Grave); en este sentido al considerar el total de caso, esto no tiene efecto directo sobre el análisis presentado. La figura 1 muestra una serie de tiempo sobre la Tasa de incidencia en todo el estado Zulia. Estos resultados revelan una mayor tasa, durante el año 2010. Se observa también, una variación estacional principalmente durante las primeras 10 semanas de los años 2008, 2014 y 2016. Además, visiblemente notoria durante los años 2014, 2010 y 2015 a partir de la semana 40 (18).

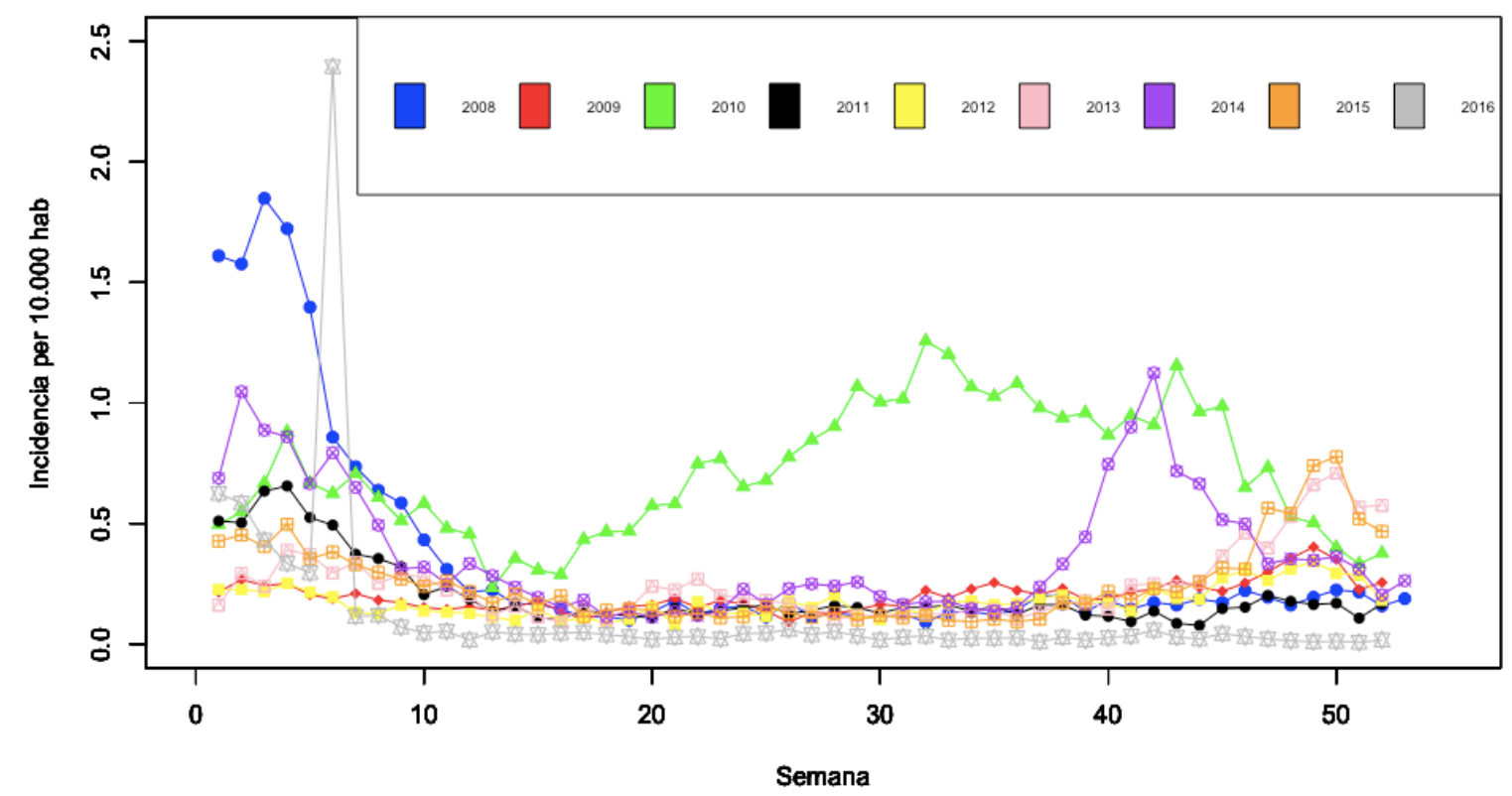

Figura 1: Distribución de la incidencia del dengue, agregada por semanas cada 10,000 habitantes en el estado Zulia, desde 2008-2016.

En los últimos años se ha observado, tanto en las regiones del sudeste asiático, como en América, cuadros atípicos de dengue con compromiso de órganos específicos como el sistema nervioso central, hígado y miocardio, asociados a mortalidad, lo que plantea la necesidad de considerar el dengue como una enfermedad aguda grave con manifestaciones multisistémicas. En áreas donde circulan virus de más de un serotipo, o en las que tienen lugar infecciones secuenciales causadas por

10 Vol. 5, núm. 3, julio 2019, pp. 3-26

Teresa Isabel Véliz Castro, Nereida Valero Cedeño, Violeta Maricela Dalgo Flores, Maritza Guadalupe Cabrera Hernández, María José Pinos Cedeño, Anyelo Alberto Duran Mojica, Luis Eduardo Gallardo Villasmil, Víctor Saldaña Núñez; Mariana Torres Portillo 
virus de diferentes serotipos, puede tener lugar la forma más severa de infección caracterizada por hemorragia y choque (20).

\section{Factores de riesgo del dengue}

La dinámica de transmisión del DENV depende de interacciones entre el ambiente, el agente, la población de huéspedes y el vector (23). Los factores determinantes de aparición del dengue grave son complejos, están relacionados con los cambios ambientales y sociales que se produjeron durante la segunda guerra mundial, los cuales favorecieron la propagación del virus y de su vector por varios países asiáticos. Posteriormente, el crecimiento acelerado de la población, la urbanización no planificada, el insuficiente abastecimiento de agua potable, la disposición inadecuada de residuos sólidos, el aumento de viajeros y de migraciones poblacionales, el deterioro de los sistemas de salud y de los programas de vigilancia y control y la pobreza han contribuido a agravar la situación epidemiológica mundial. La aparición de cepas con mayor virulencia y capacidad de transmisión, así como la circulación simultánea de varios serotipos y genotipos en una misma región, influyen en la aparición de epidemias y de dengue grave, así como otras formas de riesgo en cuanto a formas de trasmisión de la enfermedad $(1,19,25)$.

También, los factores de riesgo individuales del huésped determinan la gravedad de la enfermedad e incluyen la infección secundaria, la edad, la etnia, y posiblemente enfermedades crónicas (asma, anemias falciformes y diabetes mellitus). Los niños, en particular son menos capaces que los adultos de compensar la extravasación plasmática y en consecuencia tienen mayor riesgo de desarrollar el choque por dengue $(18,23)$.

11 Vol. 5, núm. 3, julio 2019, pp. 3-26

Teresa Isabel Véliz Castro, Nereida Valero Cedeño, Violeta Maricela Dalgo Flores, Maritza Guadalupe Cabrera Hernández, María José Pinos Cedeño, Anyelo Alberto Duran Mojica, Luis Eduardo Gallardo Villasmil, Víctor Saldaña Núñez; Mariana Torres Portillo 


\section{Manifestaciones clínicas del dengue}

La infección por DENV es una enfermedad con presentación que varía desde una forma asintomática a una sintomática, ésta se inicia con un estadio febril agudo inespecífico (fiebre indiferenciada), la cual clínicamente es indistinguible de otras infecciones virales.

De acuerdo con la guía de la OMS en 1997 se definen los criterios para fiebre por dengue (FD), Fiebre Hemorrágica por Dengue (FHD) y Síndrome de Choque por dengue. Sin embargo, en los últimos años se han publicado diversos artículos $(26,27,28)$ que cuestionan la utilidad de esta clasificación, por considerarla rígida, demasiado dependiente de resultados de laboratorio y no inclusiva de enfermos de dengue con otras formas de gravedad, tales como la afectación particular del sistema nervioso central (SCN) (encefalitis), del corazón (miocarditis) o del hígado (hepatitis grave). Tampoco era útil para el manejo clínico de los enfermos. Por tal razón, el Programa de Adiestramiento e Investigación en Enfermedades Transmisibles de la Organización Mundial de la Salud (TDR/OMS) auspició un estudio internacional, llamado Dengue Control (DENCO) (3). Fue un estudio clínico prospectivo en regiones endémicas determinando evidencias acerca de los criterios para la clasificación de dengue dentro de niveles de severidad. Se obtuvo información clínica de casi 2.000 enfermos con dengue confirmado, procedentes de siete países de dos continentes. El estudio concluyó que del 18 a 40\% de los casos no podían ser clasificados mediante la clasificación conocida y más de

12 Vol. 5, núm. 3, julio 2019, pp. 3-26

Teresa Isabel Véliz Castro, Nereida Valero Cedeño, Violeta Maricela Dalgo Flores, Maritza Guadalupe Cabrera Hernández, María José Pinos Cedeño, Anyelo Alberto Duran Mojica, Luis Eduardo Gallardo Villasmil, Víctor Saldaña Núñez; Mariana Torres Portillo 
$15 \%$ de casos con choque tampoco podían ser clasificados como casos graves de dengue, porque no cumplían con alguno de los criterios para ser considerado FHD/SCD. Los resultados del estudio confirmaron que, mediante el uso de un conjunto de datos clínicos, parámetros de laboratorio, o ambos, se puede observar una clara diferencia entre pacientes con dengue grave (DG) y dengue no grave. Sin embargo, por razones prácticas fue conveniente dividir el gran grupo de pacientes con dengue no-grave en dos subgrupos: pacientes con signos de alarma y aquellos sin éstos; teniendo muy en cuenta que los pacientes con dengue sin signos de alarma pueden desarrollar DG $(3,15,23)$.

\section{DENGUE NO GRAVE}

\section{I.1. SIN SIGNOS DE ALARMA}

Dengue probable: vivir en áreas endémicas de dengue o viaje a ella, Fiebre y dos o más de los siguientes criterios:

- $\quad$ Náuseas, vómitos

- $\quad$ Erupción cutánea

- $\quad$ Molestias y dolores

- $\quad$ Prueba de Torniquete positiva

- Leucopenia

- Cualquier signo de alarma

Dengue confirmado por laboratorio Importante, cuando no hay signos de extravasación plasmática.

\section{I.2. CON SIGNOS DE ALARMA}

13 Vol. 5, núm. 3, julio 2019, pp. 3-26 
- Dolor abdominal intenso o abdomen doloroso a la palpación

- Vómitos persistentes

- $\quad$ Acumulación clínica de líquidos

- $\quad$ Sangrado de mucosas

- Letargia o Agitación

- Dolor precordial

- $\quad$ Hepatomegalia mayor de $2 \mathrm{cms}$

- Laboratorio: Aumento del hematocrito con rápida disminución del número de plaquetas.

\section{DENGUE GRAVE}

- $\quad$ Extravasación del plasma conduce a Síndrome de Choque por Dengue, y Acumulación de líquidos con insuficiencia respiratoria

- $\quad$ Hemorragia grave

- $\quad$ Compromiso grave de órganos:

Hígado: AST o ALT superiores a 1000

Sistema Nervioso Central: Alteración de la Conciencia.

Corazón y otros órganos.

\section{Fases evolutivas del dengue}

Después del periodo de incubación, la enfermedad comienza abruptamente y es seguida por tres fases: febril, crítica y la de recuperación (3). 
La fase febril dura generalmente de 2 a 7 días, los pacientes desarrollan fiebre alta repentina, acompañada o no con exantema, malestar general, mialgias, artralgias, dolor de cabeza, de garganta, retro-orbital, anorexia, náuseas y vómitos (29). En esta fase temprana, es difícil la diferenciación de dengue con otras enfermedades; se destacan influenza y otros virus respiratorios, enfermedades febriles exantemáticas de la infancia, leptospirosis, fiebre amarilla, fiebre tifoidea, hepatitis, las púrpuras secundarias a bacteriemias, así como otras fiebres hemorrágicas virales (30). Además, son indistinguibles entre casos de dengue grave del no grave; por lo tanto, el seguimiento de los signos de alarma y de los parámetros de laboratorio son cruciales para el reconocimiento de la progresión a la fase crítica. En algunos casos se presentan hemorragias leves como petequias, epistaxis y gingivorragia. El hígado puede aumentar de tamaño. Una prueba de torniquete positiva y el inicio al tercer día de una disminución progresiva de la cuenta de leucocitos totales aumenta la probabilidad de dengue (19).

La fase crítica se inicia entre el 3er al 7mo día de evolución de la enfermedad; es el periodo donde la fiebre se presenta en menor proporción, es decir, temperaturas de $37,5-38^{\circ} \mathrm{C}$ o menores, puede ocurrir un aumento de la permeabilidad vascular en paralelo con incremento del hematocrito en un $20 \%$ o más. El tiempo clínicamente significativo de la extravasación plasmática por lo general es de 24 a 48 horas, puede detectarse derrame pleural y ascitis dependiendo del grado de escape y de volumen de rehidratación. Algunos pacientes evolucionan a la forma más grave; precedido por signos de alarma; entre ellos dolor espontáneo o durante la palpación del abdomen, vómitos persistentes, acumulación clínica de fluidos, sangrado de mucosas, letargia, irritabilidad, hepatomegalia $>2 \mathrm{~cm}$ y aumento de hematocrito asociado a una rápida caída de plaquetas (18). Puede desarrollarse un grave

\footnotetext{
15 Vol. 5, núm. 3, julio 2019, pp. 3-26

Teresa Isabel Véliz Castro, Nereida Valero Cedeño, Violeta Maricela Dalgo Flores, Maritza Guadalupe Cabrera Hernández, María José Pinos Cedeño, Anyelo Alberto Duran Mojica, Luis Eduardo Gallardo Villasmil, Víctor Saldaña Núñez; Mariana Torres Portillo
} 
deterioro orgánico como hepatitis, encefalitis o miocarditis y/o hemorragias severas sin extravasación plasmática evidente o choque (3).

El dengue grave se caracteriza por la extravasación plasmática que lleva al SCD, y/o acumulación de líquido, con o sin dificultad respiratoria y/o daño severo de órganos (hígado, SNC, corazón, entre otros) y/o hemorragias graves. Por lo general, se desencadena en los días de defervescencia (fase crítica) entre el 4to o 5to día de evolución de la enfermedad, precedido por los signos de alarma. Durante la etapa inicial del choque, los mecanismos compensatorios que mantienen normal la presión sanguínea sistólica, también producen taquicardia y vasoconstricción periférica con reducción de perfusión cutánea. La presión diastólica aumenta hacia la sistólica con pulso débil y rápido. Finalmente, existe una descompensación y ambas presiones desaparecen abruptamente. La hipotensión prolongada y la hipoxia pueden conducir a la falla de órganos y un curso clínico extremadamente difícil. Los pacientes son considerados que presentan choque si la presión de pulso (es decir la diferencia entre sistólica y diastólica) es de $\leq 20 \mathrm{~mm} \mathrm{Hg}$ en niños o si la persona presenta signos de mala perfusión capilar (extremidades frías, llenado capilar lento o pulso rápido). En los adultos, la hipotensión de $\leq 20 \mathrm{~mm} \mathrm{Hg}$ puede indicar un choque más grave, dado que está asociada con choque prolongado el cual a menudo es complicado con hemorragias mayores (23).

Cada vez con mayor frecuencia se describen las llamadas manifestaciones inusuales, de las cuales la insuficiencia hepática aguda, miocardiopatías y trastornos neurológicos (encefalitis y encefalopatía) son las observadas con mayor frecuencia. La hipotensión, edema cerebral, hemorragias focales y la insuficiencia hepática fulminante pueden ser causa de la encefalopatía $(31,32,33)$. 
Después de la etapa crítica, el enfermo pasa un tiempo variable en la etapa de recuperación, durante este período el paciente debe eliminar fisiológicamente el exceso de líquidos que se había extravasado hasta normalizar todas sus funciones vitales; en el niño y el adulto sano esta diuresis aumentada es bien tolerada, pero hay que vigilar especialmente a cardiópatas, nefrópatas o personas ancianas (23).

\section{¿Utilidad de esta clasificación clínica en áreas endémicas?}

Poco tiempo después de la primera clasificación de la enfermedad por DENV aceptada y difundida por la OMS se inicia la discusión sobre la misma; así en 1972 se produjo una epidemia por DENV-2 en una isla remota del Pacífico Sur, donde no existía evidencia de infección previa. Algunos infectados se ajustaron a la definición de FHD y se observaron 12 casos fatales; indudablemente, se trataba de una infección primaria en ausencia de anticuerpos preexistentes contra el virus que inmuno potenciaran la infección. Observaciones semejantes se efectuaron durante una epidemia en Fiji en 1975, en donde se encontró que no había diferencias significativas en la incidencia de hemorragia y otros síntomas en infecciones primarias versus infecciones secundarias. A raíz de estos datos, Rosen cuestiona la justificación de separar el dengue en dos entidades clínicas (benigna y grave), así como la validez de las definiciones de la OMS para FHD (34).

Otro estudio que rebate la inicial propuesta sobre DENV fue el realizado en Tahití, donde se examinaron los factores de riesgo para FHD, después de dos epidemias consecutivas por DENV-3 en 1989 y DENV-2 en 1996. El estudio se basó en el comportamiento de 401 niños hospitalizados por dengue, con edades variables. Diez de estos casos fueron fatales. Al utilizar la clasificación de la OMS y otros criterios clínicos y biológicos, se seleccionaron los 50 casos más graves. Diecisiete (34\%) no 
cumplieron con los requisitos para FHD, porque no se detectó aumento en la permeabilidad vascular. De estos pacientes, 6 fallecieron por trastornos hepáticos (transaminasas elevadas más de 20 veces), trombocitopenia severa, hemorragia severa y choque. El estudio demostró que muchos casos de infección con DENV no cumplen con la definición de la OMS para FHD (35).

Murgue y col., (35) publican una evaluación de la severidad del dengue en la Polinesia francesa, basada en el análisis retrospectivo de 403 casos confirmados por laboratorio. En este estudio se clasificaron los pacientes según los criterios establecidos por la OMS 1997, y además se sometieron a un proceso de análisis basado en criterios básicos clínicos y biológicos por medio del cual se asignó un score de severidad; se seleccionaron los 50 casos más severos encontrando que la disminución progresiva del contaje de plaquetas, asociado con desórdenes hepáticos, era el principal criterio de severidad y al comparar su clasificación con la propuesta por la OMS concluye que no se corresponden y proponen que la falla hepática debe ser considerada como una forma específica severa (36).

Como se mencionó anteriormente, la naturaleza compleja del sistema de clasificación de 1997 de la OMS, la frecuencia de las pruebas de laboratorio, los parámetros hematológicos, el requisito de los cuatro criterios para DH que debían cumplirse incluso si el shock se encuentra presente, y el hecho de que la evidencia de apoyo para el dengue hemorrágico se identifica a menudo sólo durante la fase de recuperación de la enfermedad fueron elementos decisivos en esta paradoja de la clasificación del dengue. A la inversa, muchos casos que cumplían todos los requisitos para el diagnóstico de dengue hemorrágico podían ser manejados de forma ambulatoria con los criterios iniciales $(37,38)$.

Independientemente de la clasificación de la enfermedad provocada por el virus dengue, la forma dengue grave se asocia a ciertas manifestaciones que incluyen hemorragia o trombocitopenia, 
alteraciones hepáticas, manifestaciones del SNC y síndrome de shock. Además, aunque el choque hipovolémico asociado a un aumento en la permeabilidad vascular es una manifestación obvia de enfermedad grave, este fenómeno puede ocurrir en ausencia de trombocitopenia o hemorragia. Muchos estudios indican que es muy difícil determinar clínicamente la fuga de plasma durante la fase aguda de la enfermedad, cuando los síntomas clínicos de efusión están ausentes y el valor del hematocrito se presenta en el rango normal. Como consecuencia y utilizando la clasificación previa de la OMS, los clínicos tienden a clasificar todos los casos graves como FHD. Esto conlleva a estimados confusos de la incidencia e incluso a conceptos erróneos sobre la patogénesis de la enfermedad. Por estas razones, se plantea que las definiciones previas de la OMS para FD y FHD son inadecuadas y desorientadoras. Se sugiere que un abordaje alternativo para la clasificación de casos podría ser "grave" vs "no grave", y que los casos graves deberían abarcar todo el rango de manifestaciones comprendiendo choque con o sin evidencia de efusión plasmática, manifestaciones de daño hepático, del SNC, hemorragia y trombocitopenia. Así, la definición de choque debido a pérdida de plasma sería simple y práctica para el diagnóstico y manejo del paciente (37).

Reconociendo estas dificultades, varios países realizaron adaptaciones locales a la clasificación de casos de 1997, introdujeron nuevas categorías que se consideraron para reflejar la clínica de los patrones de enfermedad. En Brasil, Gomes y col., (2001), realizaron un manual para la atención del paciente con dengue clasificando los 4 casos en grupos de severidad y proponiendo pautas para su adecuado tratamiento, considerando algunos conceptos clínicos y de laboratorio fundamentales para el manejo de pacientes con sospecha de dengue (38).

En Venezuela, en Barquisimeto, Finizola y Tovar (2001), crearon una escala de evaluación de severidad de pacientes con dengue, tomando en cuenta la patología y patogénesis descrita en la 
enfermedad, los signos cardinales, y los hallazgos de laboratorio propuestos por la OMS. A esa escala se le denominó, índice de severidad del estado clínico (ISEC) (39).

En un estudio realizado en el estado Zulia para describir y analizar los hallazgos anatomopatológicos relevantes en casos fatales de dengue en un área endémica venezolana se encontró que todos los casos evidenciaron enfermedad pulmonar severa (daño alveolar difuso, edema pulmonar no cardiogénico, tromboembolismo, bronconeumonía, neumonitis, hemorragia intraalveolar); 6 mostraron derrame en cavidad pleural o abdominal, o en ambas. Necrosis hepática focal se presentó en 2 casos. La causa de muerte se relacionó con insuficiencia respiratoria en 6 casos secundaria a enfermedad pulmonar, 2 con choque hipovolémico y 1 desarrolló insuficiencia hepática. Por lo que estos autores concluyeron que los casos mortales por dengue estudiados evidenciaron compromiso pulmonar severo relacionado con la muerte, a diferencia de lo reportado en otros estudios de necropsias en América. También desarrollaron afectación multiorgánica, que en la mayoría de los casos condujo a la muerte no vinculada directamente a choque hipovolémico. Estos hallazgos de necropsias permitirían proyectar estrategias terapéuticas en pro de evitar la mortalidad (20).

Poco tiempo después, en 2002 Poletto y col., (40) realizan un estudio comparativo del paciente con Fiebre Dengue, aplicando la clasificación de la OMS y el ISEC y concluyen que un porcentaje significativo $(76 \%)$ de casos de las formas menos severas consideradas por la OMS tuvieron un ISEC elevado y evolucionaron a dengue hemorrágico. En una revisión reciente, se plantea que la nueva clasificación de 2009 presenta mejoras significativas en el DF / DH / DSS en dos áreas fundamentales: en primer lugar, refleja la gravedad de la enfermedad en tiempo real, y en segundo lugar, permite la identificación de una proporción mayor de casos clínicamente severos (41). Destaca que la clasificación anterior de dengue es muy útil en el contexto de la comprensión de la fisiopatología del

20 Vol. 5, núm. 3, julio 2019, pp. 3-26 
dengue a la cual se adjudica los actuales conocimientos de la misma, así como también esta clasificación permitió comprender los mecanismos de una infección primaria y/o secundaria de dengue y sus consecuencias.

\section{Conclusión}

La primera clasificación propuesta por la OMS permitió el conocimiento actual y la comprensión de la fisiopatología de la enfermedad por DENV al tener en cuenta criterios clínicos y complementarlos con resultados de laboratorio, que aunque numéricos y cerrados sirvieron de guía para denotar progreso y estadio de la enfermedad, de esta primera clasificación, también se obtiene que el contaje plaquetario es un signo aún impreciso de gravedad de hemorragia y que pacientes con contajes plaquetarios más altos pudieran tener otros signos de alarma; en contraste, la clasificación revisada de DENV del 2009, presenta gran cantidad de signos de alarma que pudiera conllevar en áreas endémicas a hospitalizaciones numerosas e innecesarias, colapsando instituciones de salud sobre todo en países subdesarrollados como Venezuela donde aún la infraestructura es insuficiente para el total de pacientes que consultan y que ameritan su ingreso, así como también son escasos los espacios de aislamiento para pacientes con DENV. Así como en la clasificación inicial, pacientes considerados como FD que no cumplían todos los criterios para FHD y que presentaron complicaciones y cuadros clínicos graves se manejaron de forma intrahospitalaria, la última clasificación debe incluir nuevos criterios de admisión a las instituciones de salud. Actualmente, se lleva a cabo una investigación con pacientes en edad pediátrica, procedentes de Venezuela, donde se comparan criterios de ambas clasificaciones de la enfermedad, adaptabilidad a la región y pruebas de significancia que puedan sustentar la necesidad de mejoras en el sistema de clasificación actual. Un punto crucial en ambas clasificaciones, y es que a pesar de que en la primera no se consideraron otras formas de presentación

\footnotetext{
21 Vol. 5, núm. 3, julio 2019, pp. 3-26

Teresa Isabel Véliz Castro, Nereida Valero Cedeño, Violeta Maricela Dalgo Flores, Maritza Guadalupe Cabrera Hernández, María José Pinos Cedeño, Anyelo Alberto Duran Mojica, Luis Eduardo Gallardo Villasmil, Víctor Saldaña Núñez; Mariana Torres Portillo
} 
de dengue como encefalitis, miocarditis, hepatitis, la segunda también excluye los niveles de transaminasas actualmente aceptados pare definir esteatosis, necrosis o presencia de cuerpos de Councilman, estas entidades permanecen fuera de un conjunto sindromático al que se pudiera denominar otras formas de dengue severo o complicaciones del dengue, y que pudiera incluir criterios clínicos, de laboratorio e imágenes específicos para su denominación como ecocardiograma, TAC cerebral, para lograr de forma correcta la mejor definición de este síndrome.

Es importante considerar una reclasificación, como las descritas sobre manifestaciones clínicas inusuales del dengue hemorrágico en niños (42). Es imperativo que los estudios de investigación incluyan detalles suficientes en los datos clínicos para justificar sus conclusiones. A medida que el dengue continúa expandiéndose globalmente, la clasificación clínica deberá ser reexaminada y modificada. Sin embargo, no es útil aflojar los criterios para la clasificación de casos y, en particular, la incorporación de manifestaciones y mecanismos heterogéneos de la enfermedad en una clasificación única. A pesar de sus limitaciones, la clasificación de los casos de FHD ha demostrado ser útil para avanzar en observaciones importantes sobre la patogénesis de la enfermedad del dengue, como la importancia de la enfermedad secundaria.

Así mismo deben revisarse y compararse las clasificaciones actualmente en uso ya que como se mencionó en países endémicos donde existe un elevado costo por paciente con dengue, pudiera existir una demanda de hospitalizaciones innecesarias por la presencia de un solo signo de alarma en la evolución de un paciente que pudiera manejarse de forma ambulatoria, pero también porque se han descritos algunas manifestaciones inusuales en pacientes que no cumplen con criterios de gravedad y que podrían haberse manejado también erróneamente de forma ambulatoria cuando ameritan hospitalización. Se propone con este planteamiento caracterizar un complejo sindromático de

22 Vol. 5, núm. 3, julio 2019, pp. 3-26

Teresa Isabel Véliz Castro, Nereida Valero Cedeño, Violeta Maricela Dalgo Flores, Maritza Guadalupe Cabrera Hernández, María José Pinos Cedeño, Anyelo Alberto Duran Mojica, Luis Eduardo Gallardo Villasmil, Víctor Saldaña Núñez; Mariana Torres Portillo 
complicaciones en dengue, descripción y documentación de las mismas, plantear un nivel intermedio entre dengue grave y no grave con el estudio a profundidad de los signos de alarma en dengue con el propósito de optimizar el manejo y por lo tanto el pronóstico de los pacientes, continuar y profundizar en los aspectos aún no conocidos de la fisiopatología del dengue que permitirán y facilitarán la comprensión de la evolución en la enfermedad y que aún no están descritos.

Conflictos de interés: los autores declaran no tener conflictos de interés.

\section{Referencias Bibliográficas.}

1. Guzmán M., García G., y Kouri G. El dengue y el dengue hemorrágico: prioridades de investigación. Rev Panam Salud Pública. 2006; 19(3):204-214.

2. Añez G, Balza R, Valero N, Larreal Y. Impacto económico del dengue y del dengue hemorrágico en el Estado de Zulia, Venezuela, 1997-2003. Rev Panam Salud Pública. 2006; 19(5): 314-320.

3. Martínez, E. Dengue. Estudios Avanzados. 2008; 22(64): 33-52.

4. Ooi EE, Goh KT, Gubler DJ. Dengue prevention and 35 years of vector control in Singapore. Emerg Infect Dis. 2006; 12(6):887-93.

5. Shepard DS, Coudeville L, Halasa YA, Zambrano B, Dayan GH. Economic impact of dengue illness in the Americas. Am J Trop Med Hyg.2011; 84 (2): 200-7.

6. Rice, CM. Flaviviridae: The virus and their replication. Fields virology. Vol I. Trird edition. Philadephia: Lippincott-Raven Publishers; 1996. p. 931-958.

7. Chambers TJ, Hahn CS, Galler R, Rice CM. Flavivirus genome organization, expression, and replication. Annu Rev Microbiol. 1990; 44:649-88.

23 Vol. 5, núm. 3, julio 2019, pp. 3-26

Teresa Isabel Véliz Castro, Nereida Valero Cedeño, Violeta Maricela Dalgo Flores, Maritza Guadalupe Cabrera Hernández, María José Pinos Cedeño, Anyelo Alberto Duran Mojica, Luis Eduardo Gallardo Villasmil, Víctor Saldaña Núñez; Mariana Torres Portillo 
8. Holmes EC. The evolutionary biology of dengue virus. Novartis Found Symp. 2006; 277: 177-87; discussion 187-92, 251-253.

9. Monath TP. Flaviviruses. En: Virology Fields BN Editor, New York: Raven Press, p 763-814, 1996.

10. Tsai TF. Flavivirus. En: Mandell GL, Bennett JE, Dolin R. Principles and practice of infectious diseases. Fifth Edition; 2000. p. 1714.

11. Cruz A, Rolland-Burger L. El virus del dengue. Diagnóstico. 2002; 41(4): 165-172.

12. Guzmán MG; Kourí G. Dengue: an update. Lancet Infect Dis.2002; 2: 33-42.

13. Gubler, DJ. The changing epidemiology of yellow fever and dengue, 1900 to 2003: full circle? Comp Immunol Microbiol Infect Dis. 2004. 27: 319-30.

14. Valero N. Hacia el control integral del dengue. Invest Clín. 2002; 43(3):141-144.

15. PAHO. Dengue and dengue hemorrhagic fever in the Americas: guidelines for prevention and control. Washington, DC, Pan American Health Organization, No. 548. 2007.

16. Ministerio del Poder Popular para la Salud. Anuarios de Morbilidad en Venezuela 2009-2011. Disponible en:

http://www.mpps.gob.ve/index.php?option=com_phocadownload\&view=category\&id=15:anuariosde-morbilidad\&Itemid=915.

17. Ministerio del Poder Popular para la Salud. Boletín Epidemiológico 2014. Disponible en: http://www.bvs.gob.ve/boletin_epidemiologico/Boletin\%2042\%202014.pdf.

18. Cabrera M, \& Taylor G. (2019). Modelling spatio-temporal data of dengue fever using generalized additive mixed models. Spatial and spatio-temporal epidemiology, 28, 1-13.

19. Durán Mojica A, Bermúdez J, Maldonado M, Ochoa Barrientos E, Alcocer S, Levy A, Márquez A, Bermúdez I, Gómez M, Gotera J, Valero N. Incidencia y circulación del virus dengue en el estado Zulia, Venezuela (2009-2010). Ciencia Vol. 20, Nº 1 (2012) 22 - 32.

20. Arismendi Morillo G, Mauriello Rivas C, Maldonado Reverol M, Fernández Abreu M, Larreal M, Torres Nava G, et al. Correlación clínico-patológica en casos fatales de dengue en Maracaibo, Venezuela. Rev Cubana Med Trop. 63(1): 44-51.

21. Hoyos A., Pérez A. Actualización en aspectos epidemiológicos y clínicos del dengue. Revista Cubana de Salud Pública. 2010; 36(1): 149-164.

24 Vol. 5, núm. 3, julio 2019, pp. 3-26

Teresa Isabel Véliz Castro, Nereida Valero Cedeño, Violeta Maricela Dalgo Flores, Maritza Guadalupe Cabrera Hernández, María José Pinos Cedeño, Anyelo Alberto Duran Mojica, Luis Eduardo Gallardo Villasmil, Víctor Saldaña Núñez; Mariana Torres Portillo 
22. Navarrro JC, Zorrilla A y Moncada N. Primer registro de Aedes albopictus (Skuse) en Venezuela: Importancia como vector de Dengue y acciones a desarrollar. Bol Mal Salud Amb. vol.49(1): 161166.

23. Organización Mundial de la Salud. Dengue: guía para estudio, tratamiento, prevención y control, 2009.

24. Maroun S1., Marliere RC., Barcellus RC., Barbosa CN., Ramos JR., Moreira ME. Dengue infection. Case report: vertical J. Pediatr., Rio de Janeiro. 2008; 84(6): 556-9.

25. Petersen LR., Busch MP. Transfusion-transmitted arboviruses. Vox Sang.2010; 98(4): 95-503.

26. Balmaseda A, Hammond SN, Pérez AA, Cuadra R, Solano S, Rocha J, Idiaquez W, Harris E. Shot report: assessment of the world health organization scheme for classification of dengue severity in Nicaragua. Am J Trop Med Hyg.2005;73(6):1059-1062.

27. Bandyopadhyay S, Lum LC, Kroeger A. Classifying dengue: a review of the difficulties in using the WHO case classification for dengue haemorrhagic fever. Trop Med Inter Health.2006; 11(8):1238-1255 .

28. Setiati TE, Mairuhu A, Koraka P, Supriatna M, Mac Gillavry M, Bradies D, Osterhaus A, van der Meer J, van Gorp E, Soemantri A. Dengue disease severity in Indonesian children: an evaluation of the World Health Organization classification system. BMC Infect Dis. 2007; 7(22):1-8.

29. Finizola, F. Normas Básicas en el Manejo del Paciente con Fiebre Dengue, Dengue Hemorrágico y Dengue Shock. Acta Científica Venezolana.1998; 49(1): 18-24.

30. Groot H, Boshell J. Dengue, dengue hemorrágico y fiebre amarilla. Capítulo 120 en Medicina Interna, Tercera Edición (Fundación Instituto de Reumatología e Inmunología, Boehringer Ingelheim. Fernando Chalem, Jorge Escandón, Jaime Campos, Roberto Esguerra, Editores, 1998.

31. Cam BV, Fonsmark L, Hue NB, Phuong NT, Poulsen A, Heegaard ED. Prospective case-control study of encephalopathy in children with dengue hemorrhagic fever. Am J Trop Med Hyg. 2001; 65(6):848-51.

32. Nguyen TL, Nguyen TH, Tieu NT. The impact of dengue haemorrhagic fever on liver function. Res Virol.1997; 148:273-7.

33. Rigau-Perez JG. Clinical manifestations of dengue hemorrhagic fever in Puerto Rico, 1990-1991. Puerto Rico Association of Epidemiologists. Rev Panam Salud Pública. 1997; 1(5):381-8.

34. Rosen L. Dengue in Greece in 1927 and 1928 and the pathogenesis of dengue hemorrhagic fever:

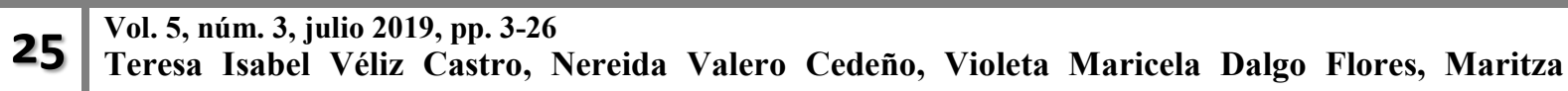
Guadalupe Cabrera Hernández, María José Pinos Cedeño, Anyelo Alberto Duran Mojica, Luis Eduardo Gallardo Villasmil, Víctor Saldaña Núñez; Mariana Torres Portillo
} 
new data and a different conclusion. Am J Trop Med Hyg 1986; 35:642-53.

35. Murgue B, Roche C, Chungue E, Deparis X. Prospective study of the duration and magnitude of viraemia in children hospitalized during the 1996-1997 dengue-2 outbreak in French Polynesia. J Med Virol 2000; 60:432-38.

36. Murgue B; Deparis. Dengue: A Polynesia based on analysis of 403 laboratory-confirmed cases. Trop Med Int Health 1999; 4(11): 765-73.

37. Corrales-Aguilar E, Hun-Opfer L. Nuevas perspectivas sobre la patogénesis del dengue. Acta Médica Costarricense. 2012. 54(2): 75-85.

38. Gomes M. Rotina para atendimiento de pacientes com dengue. Gerencia de Arbovirologia e Doenas Emergentes. Brasil. 2001.7p.

39. Finizola, F; Tovar C. Indice de Severidad del Estado Clínico en la Evaluación del paciente con Dengue. Rev. Sociedad Venezolana Puericultura y Pediatría. 2001; 64(1).

40. Poletto VJ. Evaluación Comparativa en el paciente con Fiebre Dengue aplicando la Clasificación de la Organización Mundial de la Salud y el Índice de Severidad del Estado Clínico. Hospital Pediátrico Dr. Agustín Zubillaga; 2002.

41. Jeremy J. Farrar, Tran T. Hien, Olaf Horstick, Nguyen T. Hung, Thomas Jaenisch, Thomas Junghanns, Axel Kroeger, Ida S. Laksono, Lucy Lum, Eric Martinez, Cameron P. Simmons, Adriana Tami, Kay M. Tomashek, Bridget A. Wills. Dogma in Classifying Dengue Disease. Am J Trop Med Hyg; 2013; 89(2): 198-201.

42. Stave-Salgado Karen, Herrera-Galvis Enovaldo. Manifestaciones clínicas inusuales del dengue hemorrágico en niños. Revista Ciencias Biomédicas. 2013; 4(1):69-74. 